\title{
G9a Regulates Cell Sensitivity to Radiotherapy via Histone H3 Lysine 9 Trimethylation and CCDC8 in Lung Cancer
}

\author{
Yunfen $\mathrm{Li}^{1-4, *}$ \\ Zhengting Chen $\mathbb{D}^{1}{ }^{1} *$ \\ Ke Caol,* \\ Lan Zhang'** \\ Yuhui Mal \\ Shuhui $\mathrm{Yu}^{\prime}$ \\ Hanyu Jin' \\ Xiaoling Liu' \\ Wenhui Li (D)
}

'Department of Radiotherapy, Third Affiliated Hospital of Kunming Medical, Yunnan Cancer Hospital, Kunming City,

Yunnan Province, People's Republic of

China; ${ }^{2}$ Department of Oncology, Yunnan

Boya Hospital, Kunming City, Yunnan

Province, People's Republic of China;

${ }^{3}$ College of Pharmacy, Chengdu

University of Traditional Chinese

Medicine, Chengdu City, Sichuan

Province, People's Republic of China;

${ }^{4}$ Department of Medicine, Lizhu

Pharmaceutical Group Co., Ltd., Zhuhai

City, Guangdong Province, People's

Republic of China

*These authors contributed equally to this work

\begin{abstract}
Purpose: To investigate the role and underlying mechanism of G9a and CCDC8 in lung cancer radioresistance.

Methods: Western blotting assays were used for G9a, CCDC8, H3K9me3 expression detection. MTT assays and clone formation assays were used for measuring cell proliferation activities. Flow cytometry assays were used for cell apoptosis detection. The enrichment of $\mathrm{H} 3 \mathrm{~K} 9 \mathrm{me} 3$ in $\mathrm{CCDC} 8$ promoter was measured by chromatin immunoprecipitation assay.

Results: G9a and G9a-mediated $\mathrm{H} 3 \mathrm{~K} 9 \mathrm{me} 3$ are upregulated in radioresistant lung cancer cells (A549/IR cell and XWLC-05/IR cell). Blocking G9a not only promotes radiosensitivity of A549/IR cell and XWLC-05/IR cell but also reduces aggressive behavior of radioresistant A549 cell/IR and XWLC-05/IR cell. In addition, G9a-controlled H3K9me3 is able to binding to the promoter of tumor suppressor gene CCDC8 and suppresses CCDC8 expression. CCDC8 dysregulation is responsible for G9a-mediated radioresistance of A549/IR cell and XWLC-05/IR cell.
\end{abstract}

Conclusion: G9a and $\mathrm{H} 3 \mathrm{~K} 9 \mathrm{me} 3$ contribute to the lung cancer radioresistance via modulating CCDC8 expression.

Keywords: radiotherapy, lung cancer, G9a, H3K9me3, CCDC8

\section{Introduction}

Lung cancer is the leading cause of cancer-related death worldwide, and its incidence continues to increase. ${ }^{1}$ Radiotherapy is widely used for lung cancer therapy. ${ }^{2,3}$ However, the resistance of cancer cells to radiotherapy is one of the major factors that closely related to the unsatisfactory outcome of lung cancer treatment. Thus, more efforts are required to explore the mechanism underlying lung cancer radioresistance.

CCDC8 is identified a tumor suppressor and plays a significant role in cancer development. Jiang et al suggested that CCDC8 inhibits the invasion and migration of non-small cell lung cancer cells. ${ }^{4}$ Pan indicated that CCDC8 is involved in cancer progression via interacting with other proteins. ${ }^{5}$ Recent study showed that CCDC8 contributes to the cancer therapy. For example, Nakagawa et al demonstrated $\mathrm{CCDC} 8$ contributes to gastric cancer chemotherapy resistant. ${ }^{6}$ However, the function of $\mathrm{CCDC} 8$ in lung cancer radioresistance remains obscure.

Recently, more efforts have been made to analyze the epigenetic modification, such as DNA or RNA methylation and histone methylation, of tumor-related genes. The epigenetic modification of oncogenes affects gene transcription, which results
Department of Radiotherapy, Third Affiliated Hospital of Kunming Medical, Yunnan Cancer Hospital, No.519

Kunzhou Road, Kunming City, Yunnan

Province, People's Republic of China

Email wenhuili2014@I63.com 
in altered cancer radioresistance. ${ }^{7}$ Xiang et al suggested that RNA modification play a role in cancer radiotherapy. ${ }^{8}$ Zhu et al showed DNA methylation in genes related to DNA repair and cell proliferation during radiation. ${ }^{9}$ DNA hypermethylation and histone deacetylation-mediated SOCS silencing affects cervical cancer radiotherapy. ${ }^{10}$ G9a functions as a histone methyltransferase, catalyzing the histones $\mathrm{H} 3 \mathrm{~K} 9$ trimethylation (H3K9me3). Aberrant expression of G9a contributes to lung cancer progression. ${ }^{11,12}$ Accumulating evidence demonstrated that G9a-mediated $\mathrm{H} 3 \mathrm{~K} 9$ me3 modulates gene repression during cancer development and therapy. ${ }^{13-15}$ In addition, a study indicated that $\mathrm{CCDC} 8$ is epigenetically dysregulated in breast cancer. ${ }^{16} \mathrm{Lu}$ et al suggested that in esophageal squamous cell carcinoma, CCDC 8 could be methylated. ${ }^{17}$ Thus, we hypothesis that G9a-controlled H3K9me3 modulates lung cancer radioresistance via CCDC8.

In this study, we found that the expression of G9a and $\mathrm{H} 3 \mathrm{~K} 9 \mathrm{me} 3$ are upregulated after radiation treatment in lung cancer cells. Moreover, G9a and H3K9me3 are increased in radioresistant lung cancer cells. We also observed G9a inhibit CCDC8 expression via regulating the enrichment of $\mathrm{H} 3 \mathrm{~K} 9 \mathrm{me} 3$ on $\mathrm{CCDC} 8$ promoter. And CCDC8 is responsible for $\mathrm{G} 9 \mathrm{a}$-mediated lung cancer radioresistance. These results demonstrate that G9a and CCDC 8 are promising targets for lung cancer radiotherapy.

\section{Materials and Methods}

\section{Cell Culture}

A549 cell and XWLC-05 cell were purchased from the cell bank of the Institute of Biochemistry and Cell Biology, Chinese Academy of Sciences. Cells were maintained in minimum essential medium (MEM). The media were supplemented with $10 \% \mathrm{FBS}$, penicillin $(100 \mathrm{U} / \mathrm{mL})$, and streptomycin $(100 \mathrm{mg} / \mathrm{mL})$ in a humidified atmosphere containing $5 \% \mathrm{CO}_{2}$ at $37^{\circ} \mathrm{C}$. The radioresistant A549/IR and XWLC-05/IR cell lines were obtained after repeated irradiation.

\section{Cell Transfection}

Stable knockdown of G9a and CCDC8 was achieved by RNA interference. The short hairpin RNAs (shRNAs) targeting G9a and corresponding control (termed as shNC), CCDC8 siRNA (siCCDC8) and corresponding control (termed as siNC) synthesized by GenePharma (Shanghai, China). Cells $\left(1 \times 10^{6}\right)$ were transfected using
Lipofectamine 3000 according to the manufacturer's instructions (Invitrogen, U.S.A).

\section{Chromatin Immunoprecipitation (ChIP) Assay}

The ChIP experiment was performed to determine the enrichment of $\mathrm{H} 3 \mathrm{~K} 9 \mathrm{me} 3$ on CCDC8 promoter. The A549 cells and XWLC-05 cells were crosslinked with $1 \%$ formaldehyde and quenched with $0.125 \mathrm{M}$ glycine. The collected cells were washed and lysed in lysis buffer. The samples were sheared by sonication and incubated with H3K9me3 and $\mathrm{H} 3$ antibodies for each chromatin IP experiment. The purified DNA was quantitated by qPCR with specific primers. The primer used in this study was as follows: CCCTGATCAGCCTCGATGTC, ATCAACTGG GCCTCCTTTCG.

\section{Cell Viability Assay}

Cells were seeded in 96-well plates at a density of $2.5 \times 10^{4}$ cells per well and cultured with $5 \% \mathrm{CO}_{2}$ in a $37^{\circ} \mathrm{C}$ incubator for $2 \mathrm{~h}$ to allow cells to adhere. The cisplatin was added with different concentration. After $48 \mathrm{~h}$, cell viability was measured by 3-(4,5)-dimethylthiahiazo (-z-y1)-3,5-diphenytetrazoliumromide (MTT, Beyotime, China) assay.

\section{Flow Cytometry}

Cell apoptosis was measured by flow cytometry. Indicated cells were collected and resuspended. And incubation with $5 \mu \mathrm{L}$ Annexin V-FITC and $5 \mu \mathrm{L}$ PI (BD Biosciences, USA) for $15 \mathrm{~min}$ in the dark. Apoptosis cells were detected by flow cytometry using a Flow Cytometer (Becton Dickinson, FACSCalibur).

\section{Colony Formation Assay}

A549 cells, XWLC-05 cells, A549/IR cells and XWLC05/IR cells were cultured in complete medium and plated in $10-\mathrm{cm}$ culture dishes for 2 weeks. $0.1 \%$ crystal violet was used for colonies staining.

\section{Western Blotting}

Protein concentrations were determined using the Pierce BCA Protein Assay Kit (Pierce, Rockford, IL, USA). After separation by $10 \%$ SDS-polyacrylamide gel electrophoresis (SDS-PAGE), proteins were transferred onto polyvinylidene difluoride membranes (PVDF, Millipore, Burlington, Massachusetts, USA) for $120 \mathrm{~min}$ Then, the cells were blocked with TBST containing 5\% nonfat 
skimmed milk and incubated with primary antibodies (anti-CCDC8, ab235780, 1:2000, Abcam; anti-GAPDH, ab9485, 1:5000; Abcam; anti-H3, ab1791, 1:8000, Abcam; anti-H3K9me3, ab8898, 1:5000, Abcam; antiG9a, ab185050, 1:2000, Abcam). Subsequently, secondary antibodies were added to culture the cells (goat anti-rabbit IgG H\&L, ab6721, 1:10,000; goat anti-mouse IgG H\&L, ab6789, 1:2000; Abcam). The signal detection was conducted by means of an ECL system (Life Technology, USA). GAPDH and $\mathrm{H} 3$ was regarded as an internal reference.

\section{Statistical Analysis}

The measured parameters are presented as the means \pm SD. GraphPad Prism 7.0 (La Jolla, CA, USA) was used for statistical analysis. Statistical tests for data analysis were two-tailed $t$-test and one-way ANOVA. $\mathrm{P}<0.05$ indicated a statistically significant difference. All experiments were performed in triplicate.

\section{Results}

\section{Characterization of Irradiation-Resistant Lung Cancer Cells}

In order to explore the mechanism of irradiation resistance, the irradiation-resistant A549 cell line (A549/IR) and XWLC-05 cell line (XWLC-05/IR) was generated. As shown in Figure 1A, the cell proliferation activity of A549/IR and XWLC-05/IR cell remained a higher level compared to A549 and XWLC-05 cell upon ionizing radiation. In addition, the radioresistant ability in A549/IR and XWLC-05/IR cell was increased according to clone formation assay and cell apoptosis assay (Figure 1B and C).

\section{G9a Contributes Irradiation Resistance of Lung Cancer Cells}

A study demonstrated that the expression of histone methyltransferase G9a and G9a-mediated H3K9me3 was induced under radiation treatment in lung cancer. ${ }^{18}$ In order to further identify the function of G9a in irradiation resistance of lung cancer cells, the expression of G9a and H3K9me3 was measured. As shown in Figure 2A, G9a and $\mathrm{H} 3 \mathrm{~K} 9 \mathrm{me} 3$ expression level was induced in A549/IR and XWLC-05/IR cell. We next established A549/IR and XWLC-05/IR cell stably expressing short hairpin RNAs (shRNAs) targeting G9a (shG9a). The expression of G9a and $\mathrm{H} 3 \mathrm{~K} 9 \mathrm{me} 3$ in shG9a-transfected A549/IR and XWLC-05/IR cell was decreased compared to control transfection (shNC) (Figure 2B). After ionizing radiation administration, cell proliferation rate, clone formation ability and cell apoptosis were measured (Figure 2C-E), which indicated the role of G9a in enhancing irradiation resistance in lung cancer cells.

\section{CCDC8 is a Downstream Target of G9a}

Previous study indicated that pro-apoptotic gene CCDC8 functioned as a tumor suppressor in lung cancer development. ${ }^{4}$ However, the role and possible mechanisms of CCDC8 in radiotherapy resistance of lung cancer has not been elucidated. The regulation of G9a mediated-H3K9me3 on target gene was involved in the radiotherapy of lung cancer. ${ }^{18}$ It was reported that the promoter of CCDC8 could be methylated ${ }^{19}$ and CCDC8 expression could be modulated by histone lysine demethylases via H3K9 methylation. ${ }^{6}$ Thus, we hypothesized that G9a may regulate CCDC8 expression via methylation. The expressions of CCDC8 in A549/IR cell and XWLC-05/IR cell were decreased compared to A549 cells and XWLC-05 cell (Figure 3A). After silencing G9a in A549/IR cell and XWLC-05/IR cell, the expression of CCDC8 was induced (Figure 3B). To determine the correction between G9a-mediated H3K9me3 and CCDC8, the ChIP assay was performed. Inhibition of G9a attenuated the enrichment level of $\mathrm{H} 3 \mathrm{~K} 9 \mathrm{me} 3$ on CCDC8 promoters in A549/IR cell and XWLC-05/IR cell (Figure $3 \mathrm{C}$ ). These results suggested that G9a-mediated $\mathrm{H} 3 \mathrm{~K} 9 \mathrm{me} 3$ inhibits CCDC8 via binding to the promoter.

\section{G9a and H3K9me3 Modulates Lung Cancer Cells Resistance to Radiotherapy via Modulating CCDC8}

To confirm the contribution of CCDC8 in G9a-mediated radiotherapy resistance, $\mathrm{CCDC} 8$ siRNAs (siCCDC8) and corresponding control (siNC) were transfected to shG9aA549/IR cell and shG9a-XWLC-05/IR cell (Figure 4A). Inhibition of CCDC8 abolished the effect of G9a silencing on cell proliferation activity (Figure 4B), cell colon formation ability (Figure 4C) and cell apoptosis (Figure 4D). These results demonstrated that $\mathrm{CCDC} 8$ was responsible for G9amediated lung cancer cells resistance to radiotherapy.

\section{Discussion}

In the present study, we demonstrated that G9a and G9acontrolled $\mathrm{H} 3 \mathrm{~K} 9 \mathrm{me} 3$ promote lung cancer cells resistance to radiotherapy. G9a-catalyzed $\mathrm{H} 3 \mathrm{~K} 9 \mathrm{me} 3$ reduces $\mathrm{CCDC} 8$ expression via binding to the promoter in lung cancer. 
A
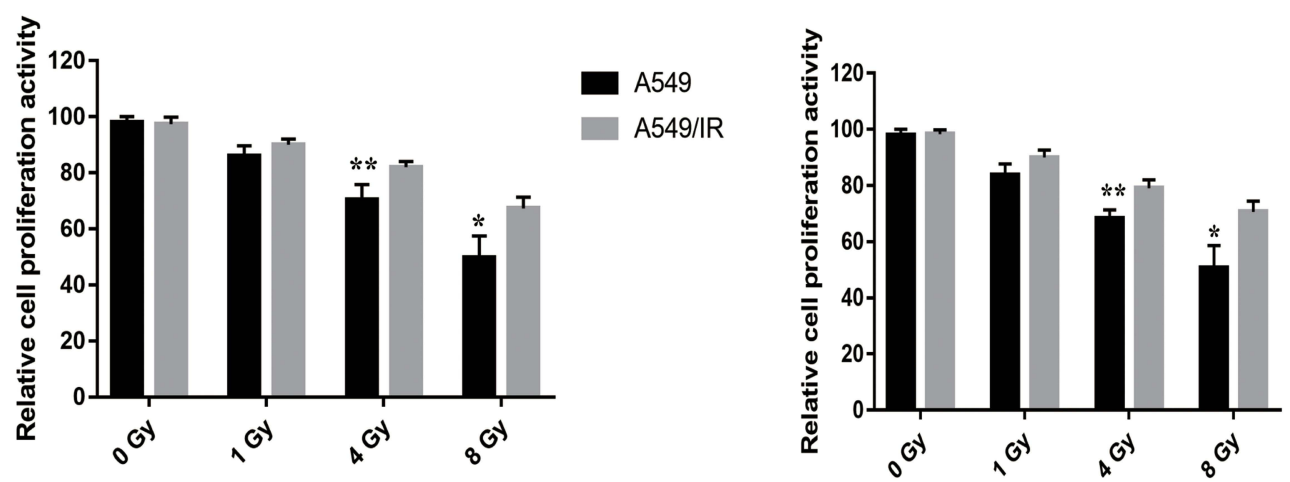

XWLC-05

A549/IR

XWLC-05/R

B

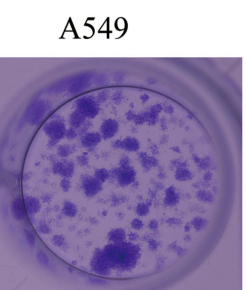

A549/IR
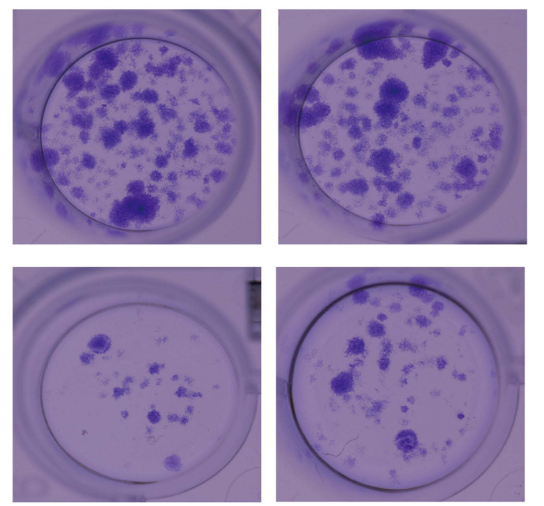

XWLC-05
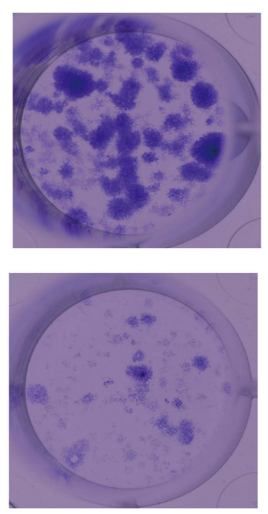

0 Gy

4 Gy
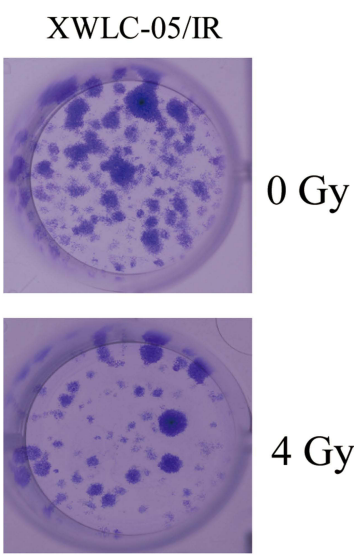

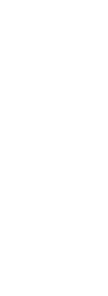

C
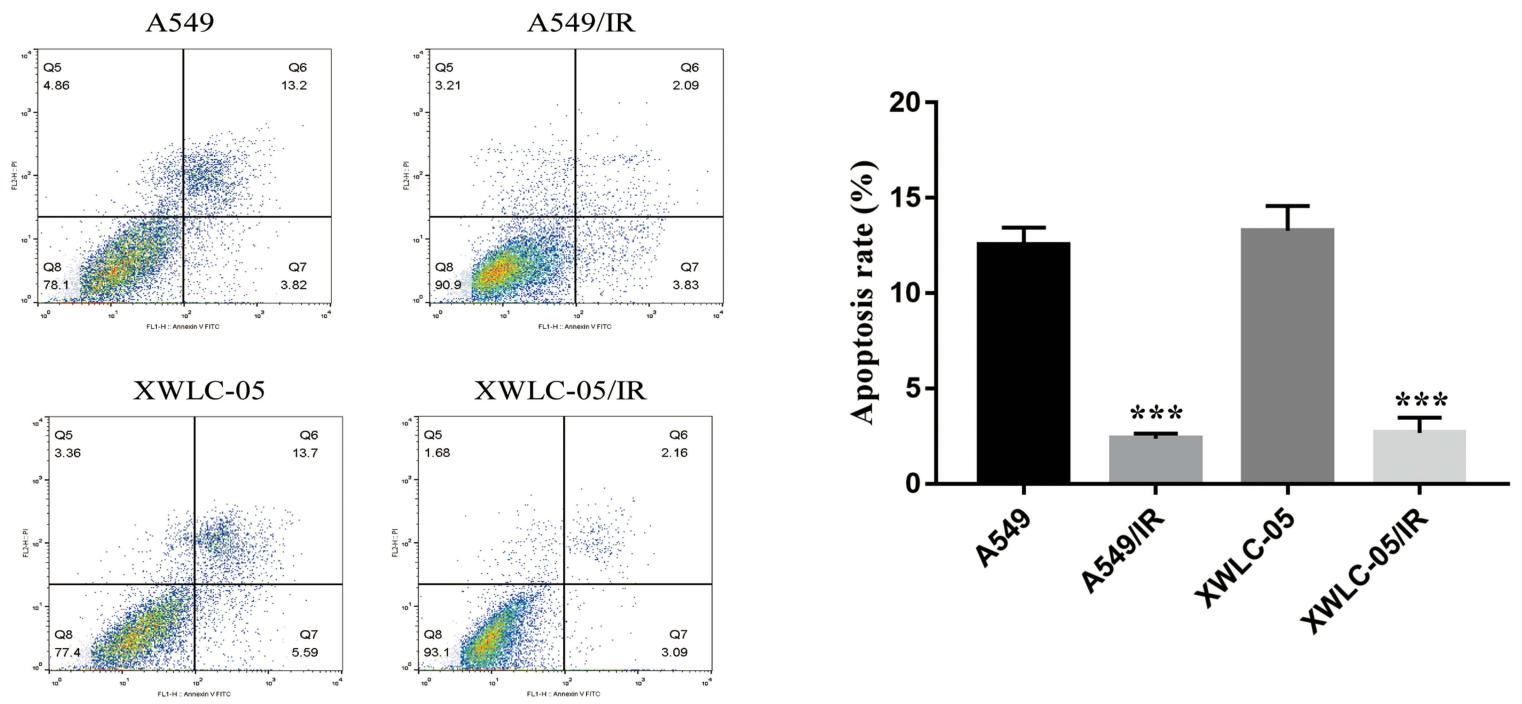

Figure I Characterization of irradiation-resistant lung cancer cells. (A) The proliferation activity of A549/IR cell, XWLC-05/IR cell, A549 cell and XWLC-05 cell was measured by MTT assay. ${ }^{*} p<0.05$, **p $<0.01$. (B) Cell viability of A549/IR cell, XWLC-05/IR cell, A549 cell and XWLC-05 cell was detected with clone formation assay. (C) Apoptosis rate of A549/IR cell, XWLC-05/IR cell, A549 cell and XWLC-05 cell was measured by flow cytometry. $* * * p<0.001$. Dates were presented as mean \pm SD, the $P$ value was determined by two-tailed $t$-test and one-way ANOVA. 
A
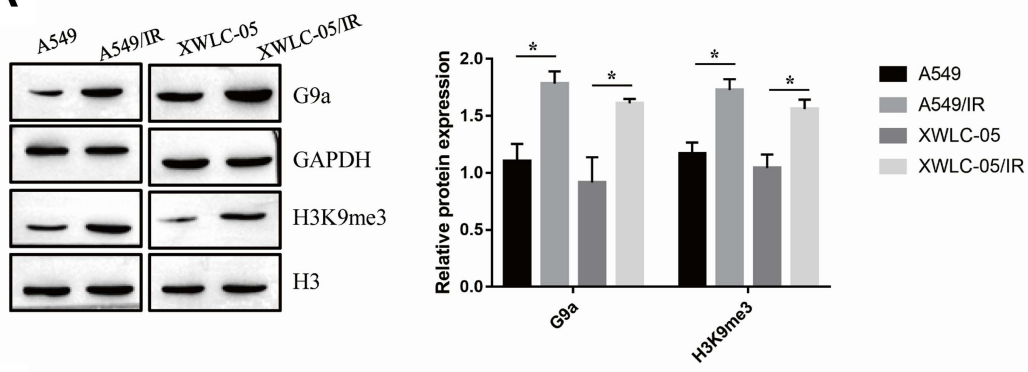

B
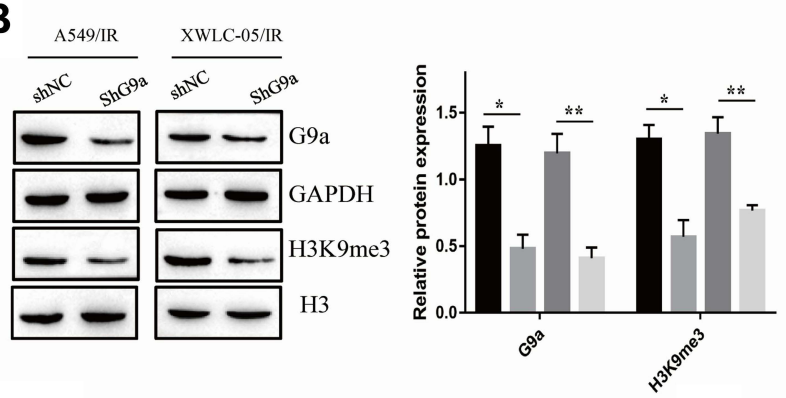

A549/IR-shNC

- A549/IR-shG9a

XWLC-05/RR-shNC

XWLC-05/IR-shG9a

C

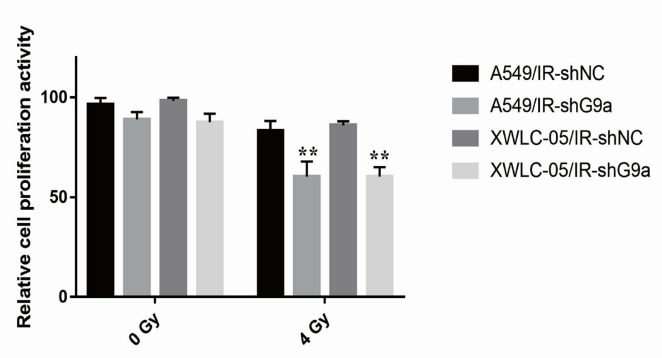

D
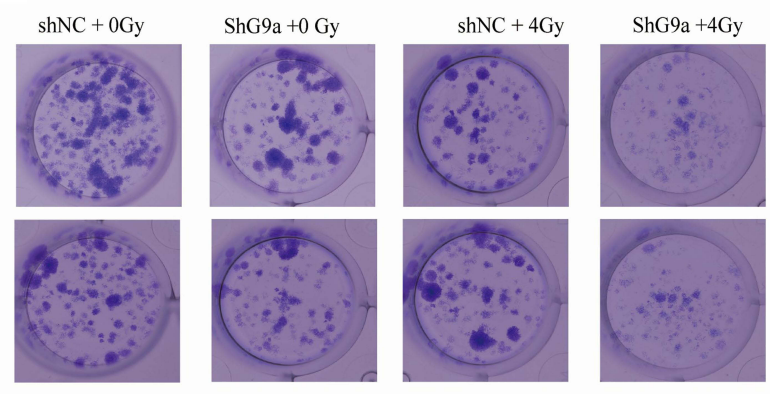

A549/IR

XWLC-05/IR

E
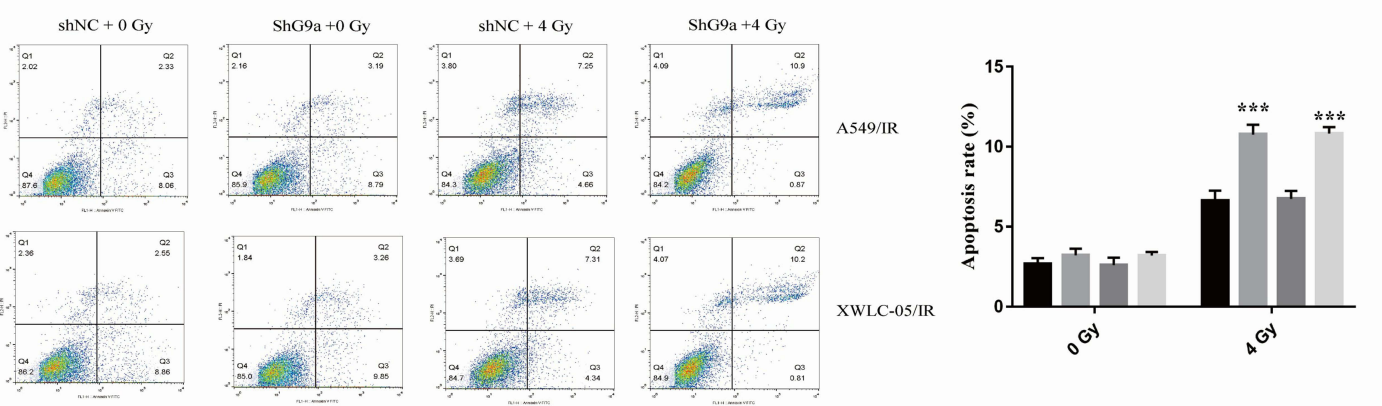

A549/IR-shNC

A549/IR-shG9a

XWLC-05/IR-shNC

XWLC-05/IR-shG9a

Figure 2 G9a contributes irradiation resistance of lung cancer cells. (A) Expression of G9a and H3K9me3 in A549/IR cell, XWLC-05/IR cell, A549 cell and XWLC-05 cell. Protein quantitative analysis was achieved by Image J. ${ }^{*} p<0.05$. (B) Expression of G9a and H3K9me3 in shG9a transfected A549/IR cell and XWLC-05/IR cell. Protein quantitative analysis was achieved by Image J. ${ }^{*} p<0.05,{ }^{*} *_{p}<0.0$ I. (C) The proliferation activity of shG9a transfected A549/IR cell and XWLC-05/IR cell was measured by MTT assay. **p < 0.0I. (D) Clone formation of shG9a transfected A549/IR cell and XWLC-05/IR cell after administrated to 0Gy and 4Gy. (E) Apoptosis rate of shG9a transfected A549/IR cell and XWLC-05/IR cell after administrated to 0Gy and 4Gy was measured by flow cytometry. $* * * p<0.00 \mathrm{I}$. Dates were presented as mean \pm SD, the $P$ value was determined by two-tailed $t$-test and one-way ANOVA.

Importantly, our results further suggested that CCDC8 is critical for G9a-mediated lung cancer cell radioresistance. Our study describes novel roles of G9a and CCDC8 in lung cancer cell radioresistance.
Studies have demonstrated that abnormal expression of G9a is linked to cancer development. Inhibition of G9a can induce bladder cancer cell apoptosis. ${ }^{20}$ In gastric cancer, G9a enhanced the cell proliferation and attenuated cell 
A
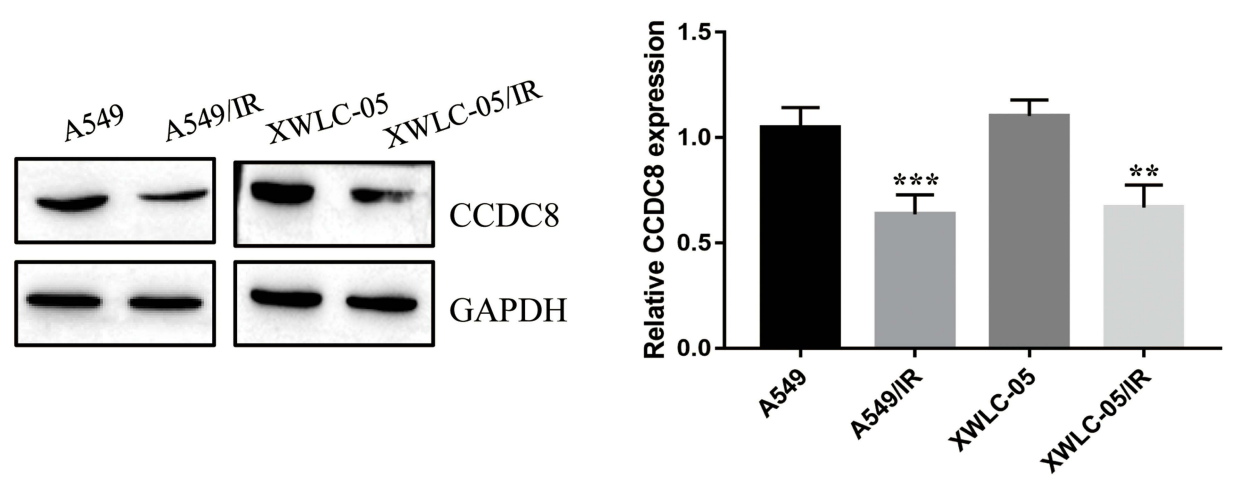

B

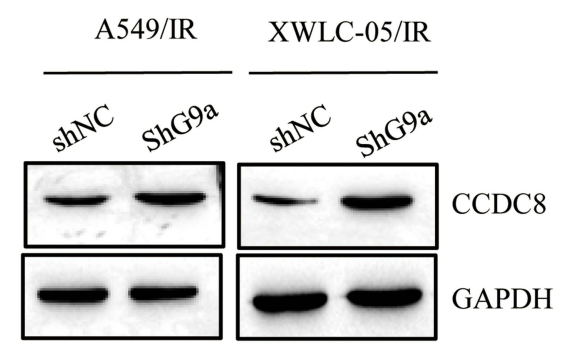

C

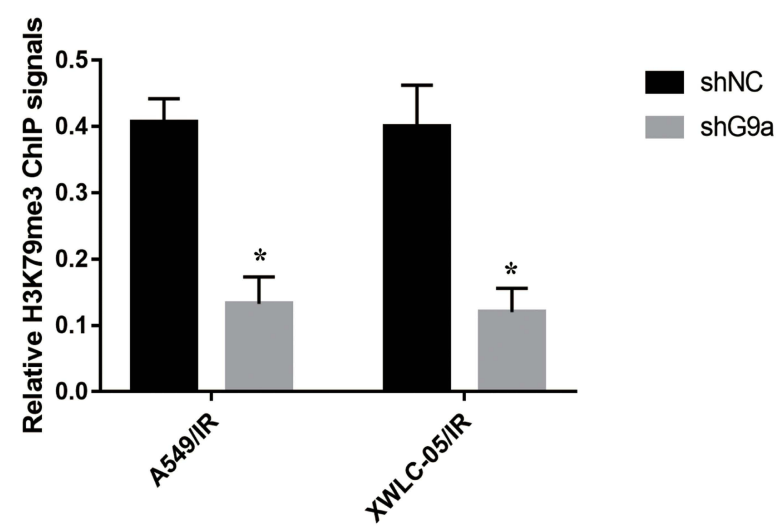

Figure 3 CCDC8 is a downstream target of G9a. (A) Expression of CCDC8 in A549/R cell, XWLC-05/IR cell, A549 cell and XWLC-05 cell was determined by Western blot assay. Protein quantitative analysis was achieved by Image J. $* * p<0.01$, ***p $<0.001$. (B) Protein expression of CCDC8 in shG9a transfected A549/IR cell and XWLC05/IR cell was determined by Western blot assay. Protein quantitative analysis was achieved by Image J. ${ }^{*} p<0.05, * * p<0.01$. (C) Enrichment of $\mathrm{H} 3 \mathrm{~K} 9 \mathrm{me} 3$ on CCDC8 promoter was determined buy ChIP assay. ${ }^{*} p<0.05$. Dates were presented as mean $\pm S D$, the $P$ value was determined by two-tailed $t$-test and one-way ANOVA.

autophagy. ${ }^{21}$ The role of G9a in cancer therapy has been well characterized. Luo et al suggested that G9a affected radio resistance of colon cancer cells. ${ }^{22}$ Pan et al suggested that G9a could be a therapeutic target in gemcitabine therapy. ${ }^{23}$ Most importantly, G9a functions as a histone methyltransferase, is responsible for catalyzing $\mathrm{H} 3 \mathrm{~K} 9 \mathrm{me} 3$.

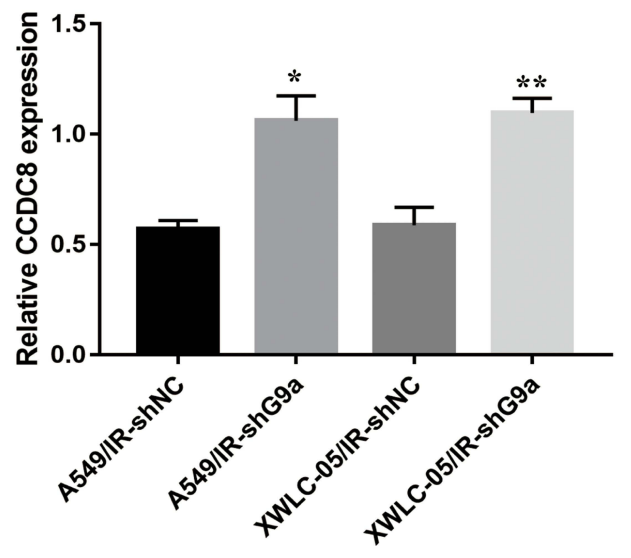

G9a-mediated H3K9me3 can modulate tumor-related genes repression during cancer development such as breast cancer $^{24}$ and glioma cancer. ${ }^{25}$ In addition, H3K9me3 served as a significant factor for cancer chemoresistance. $^{26}$ Our data showed that G9a and H3K9me3 were upregulated in in radioresistant lung 
A
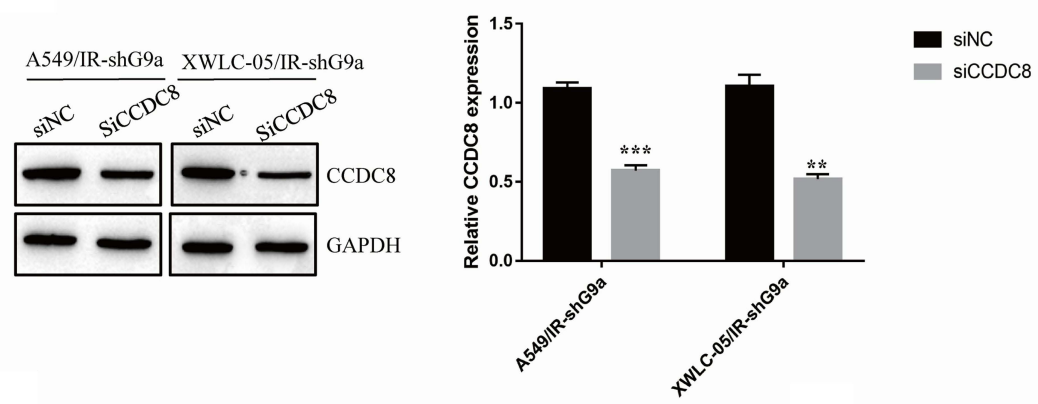

B

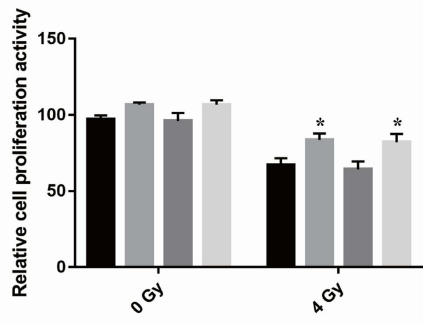

C

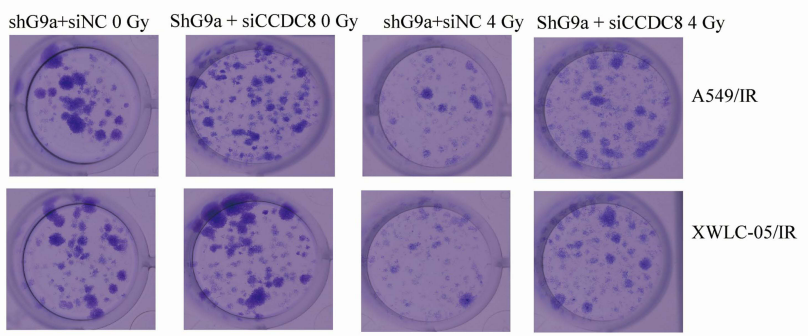

D
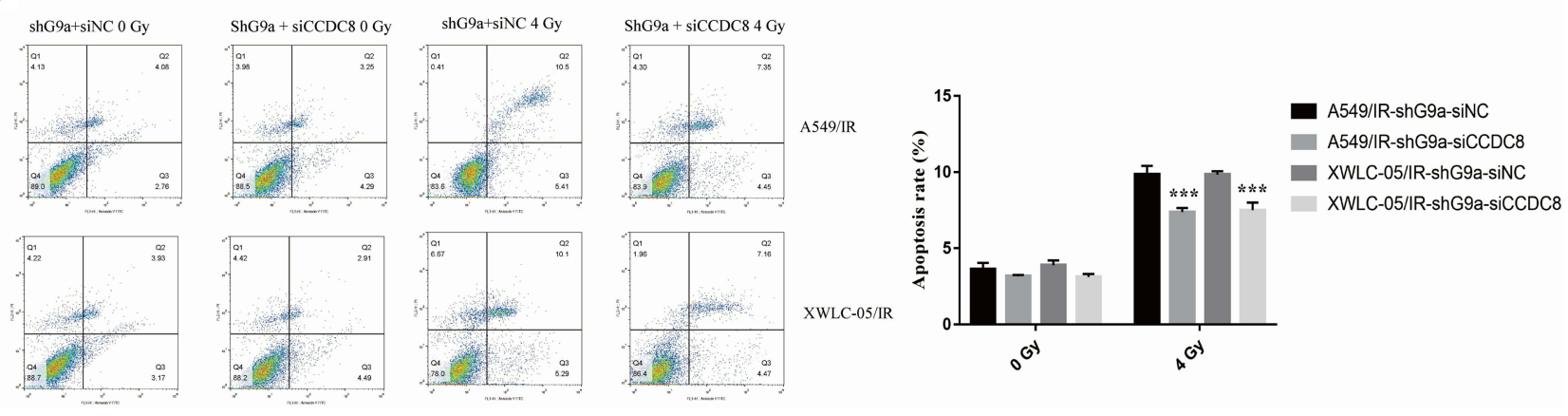

Figure $4 \mathrm{G9a}$ and $\mathrm{H} 3 \mathrm{~K} 9 \mathrm{me} 3$ modulates lung cancer cells resistance to radiotherapy via modulating CCDC8. (A) Expression of CCDC8 in A549/IR cell and XWLC-05/IR cell transfected with shG9a was measured by Western blot assay. Protein quantitative analysis was achieved by Image J. **p $<0.0 \mathrm{I}$, *** $<0.00 \mathrm{I}$. (B) The proliferation activity of siCCDC8 or siNC transfected shG9a-A549/IR cell and shG9a-XWLC-05/IR cell was determined by MTT assay. *p < 0.05 . (C) Clone formation of siCCDC8 or siNC transfected shG9a-A549/IR cell and shG9a-XWLC-05/IR cell cell after administrated to 0Gy and 4Gy. (D) Apoptosis rate of siCCDC8 or siNC transfected shG9a-A549/IR cell and shG9a-XWLC-05/IR cell was measured by flow cytometry. $* * * p<0.001$. Dates were presented as mean \pm SD, the $P$ value was determined by two-tailed $t$-test and one-way ANOVA.

cancer cells. Inhibition of G9a not only reduced $\mathrm{H} 3 \mathrm{~K} 9 \mathrm{me} 3$ level in lung cancer cells but also suppressed aggressive behavior of radioresistant lung cancer cells.

CCDC8 is identified a tumor suppressor in several types of cancer including lung cancer. ${ }^{4} \mathrm{CCDC} 8$ has been involved in gastric cancer chemotherapy. ${ }^{6}$ Accumulating studies suggested CCDC8 can be modulated via histone methylation, ${ }^{6,16}$ thus, we speculated CCDC 8 is regulated by G9a-controlled H3K9me3. In our study, a decreased level of CCDC8 was observed in radioresistant lung cancer cells. CCDC8 expression overexpression partly rescued aggressive behavior of radioresistant lung cancer cells. Most importantly, our ChIP assay showed that H3K9me3 was bound directly to the CCDC8 promoter region and inhibited its expression. We also revealed that
CCDC8 is responsible for G9a-mediated radioresistance of lung cancer cells.

\section{Conclusion}

In summary, we find G9a is upregulated in radioresistant lung cancer and promotes aggressive behavior of radioresistant cells. We also uncover the mechanism by which G9a promotes lung cancer radioresistance via modulating H3K9me3 enrichment on CCDC8 promoter.

\section{Funding}

Yunnan Provincial Science and Technology Department Kunming medicine joint project (2019FE001-154). 


\section{Disclosure}

The authors declare that they have no competing interests.

\section{References}

1. Bray F, Ferlay J, Soerjomataram I, Siegel R, Torre L, Jemal A. Global cancer statistics 2018: GLOBOCAN estimates of incidence and mortality worldwide for 36 cancers in 185 countries. CA Cancer J Clin. 2018;68(6):394-424. doi:10.3322/caac.21492

2. Ma L, Qiu B, Zhang J, et al. Survival and prognostic factors of non-small cell lung cancer patients with postoperative locoregional recurrence treated with radical radiotherapy. Chin J Cancer. 2017;36 (1):93. doi:10.1186/s40880-017-0261-0

3. Hu X, Bao Y, Xu Y, et al. Final report of a prospective randomized study on thoracic radiotherapy target volume for limited-stage small cell lung cancer with radiation dosimetric analyses. Cancer. 2020;126 (4):840-849. doi:10.1002/cncr.32586

4. Jiang G, Zhang X, Zhang Y, et al. Coiled-coil domain-containing protein 8 inhibits the invasiveness and migration of non-small cell lung cancer cells. Hum Pathol. 2016;56:64-73. doi:10.1016/j. humpath.2016.06.001

5. Pan ZQ. Cullin-RING E3 Ubiquitin Ligase 7 in Growth Control and Cancer. Adv Exp Med Biol. 2020;1217:285-296. doi:10.1007/978981-15-1025-0 17

6. Nakagawa T, Sato Y, Tanahashi T, et al. JMJD2A sensitizes gastric cancer to chemotherapy by cooperating with CCDC8. Gastric Cancer. 2020;23(3):426-436. doi:10.1007/s10120-019-01024-9

7. Smits K, Melotte V, Niessen H, et al. Epigenetics in radiotherapy: where are we heading? Radiother Oncol. 2014;111(2):168-177. doi:10.1016/j.radonc.2014.05.001

8. Xiang M, Liu W, Tian W, You A, Deng D. RNA N-6-methyladenosine enzymes and resistance of cancer cells to chemotherapy and radiotherapy. Epigenomics. 2020;12(9):801-809. doi:10.2217/epi-2019-0358

9. Zhu X, Wang Y, Tan L, Fu X. The pivotal role of DNA methylation in the radio-sensitivity of tumor radiotherapy. Cancer Med. 2018;7 (8):3812-3819. doi:10.1002/cam4.1614

10. Kim M, Kim M, Kim W, et al. Suppressor of cytokine signaling (SOCS) genes are silenced by DNA hypermethylation and histone deacetylation and regulate response to radiotherapy in cervical cancer cells. PLoS One. 2015;10(4):e0123133. doi:10.1371/journal.pone.0123133

11. Rowbotham S, Li F, Dost A, et al. H3K9 methyltransferases and demethylases control lung tumor-propagating cells and lung cancer progression. Nat Commun. 2018;9(1):4559. doi:10.1038/s41467-018-07077-1

12. Chen M, Hua K, Kao H, et al. H3K9 histone methyltransferase G9a promotes lung cancer invasion and metastasis by silencing the cell adhesion molecule Ep-CAM. Cancer Res. 2010;70(20):7830-7840. doi:10.1158/0008-5472.can-10-0833

13. Dang N, Jiao J, Meng X, An Y, Han C, Huang S. Abnormal overexpression of G9a in melanoma cells promotes cancer progression via upregulation of the Notch1 signaling pathway. Aging. 2020;12 (3):2393-2407. doi:10.18632/aging. 102750
14. Tu W, Shiah Y, Lourenco C, et al. MYC interacts with the G9a histone methyltransferase to drive transcriptional repression and tumorigenesis. Cancer Cell. 2018;34(4):579-595.e8. doi:10.1016/j. ccell.2018.09.001

15. Lai Y, Chen J, Tsai H, Chen T, Hung W. The SUV39H1 inhibitor chaetocin induces differentiation and shows synergistic cytotoxicity with other epigenetic drugs in acute myeloid leukemia cells. Blood Cancer J. 2015;5(5):e313. doi:10.1038/bcj.2015.37

16. Pangeni R, Channathodiyil P, Huen D, et al. The GALNT9, BNC1 and $\mathrm{CCDC} 8$ genes are frequently epigenetically dysregulated in breast tumours that metastasise to the brain. Clin Epigenetics. 2015;7(1):57. doi:10.1186/s13148-015-0089-x

17. Lu T, Chen D, Wang $\mathrm{Y}$, et al. Identification of DNA methylation-driven genes in esophageal squamous cell carcinoma: a study based on The Cancer Genome Atlas. Cancer Cell Int. 2019;19(1):52. doi:10.1186/s12935-019-0770-9

18. Nagaraja S, Subramanian U, Nagarajan D. Radiation-induced H3K9 methylation on E-cadherin promoter mediated by ROS/Snail axis: role of G9a signaling during lung epithelial-mesenchymal transition. Toxicol in Vitro. 2021;70:105037. doi:10.1016/j.tiv.2020.105037

19. Morris M, Ricketts C, Gentle D, et al. Genome-wide methylation analysis identifies epigenetically inactivated candidate tumour suppressor genes in renal cell carcinoma. Oncogene. 2011;30 (12):1390-1401. doi:10.1038/onc.2010.525

20. Cao Y, Sun J, Li M, et al. Inhibition of G9a by a small molecule inhibitor, UNC0642, induces apoptosis of human bladder cancer cells. Acta Pharmacol Sin. 2019;40(8):1076-1084. doi:10.1038/ s41401-018-0205-5

21. Yin C, Ke X, Zhang R, et al. G9a promotes cell proliferation and suppresses autophagy in gastric cancer by directly activating mTOR. FASEB J. 2019;33(12):14036-14050. doi:10.1096/fj.201900233RR

22. Luo C, Wang J, Hung W, et al. G9a governs colon cancer stem cell phenotype and chemoradioresistance through PP2A-RPA axis-mediated DNA damage response. Radiother Oncol. 2017;124 (3):395-402. doi:10.1016/j.radonc.2017.03.002

23. Pan M, Hsu M, Luo C, Chen L, Shan Y, Hung W. The histone methyltransferase G9a as a therapeutic target to override gemcitabine resistance in pancreatic cancer. Oncotarget. 2016;7 (38):61136-61151. doi:10.18632/oncotarget.11256

24. Crawford N, McIntyre A, McCormick A, D'Costa Z, Buckley N, Mullan P. TBX2 interacts with heterochromatin protein 1 to recruit a novel repression complex to EGR1-targeted promoters to drive the proliferation of breast cancer cells. Oncogene. 2019;38 (31):5971-5986. doi:10.1038/s41388-019-0853-z

25. Guo A, Huang Y, Ma X, Lin R. Mechanism of G9a inhibitor BIX-01294 acting on U251 glioma cells. Mol Med Rep. 2016;14 (5):4613-4621. doi:10.3892/mmr.2016.5815

26. Paschall A, Yang D, Lu C, et al. H3K9 trimethylation silences fas expression to confer colon carcinoma immune escape and 5-fluorouracil chemoresistance. J Immunol. 2015;195(4):1868-1882. doi:10.4049/jimmunol.1402243
OncoTargets and Therapy

\section{Publish your work in this journal}

OncoTargets and Therapy is an international, peer-reviewed, open access journal focusing on the pathological basis of all cancers, potential targets for therapy and treatment protocols employed to improve the management of cancer patients. The journal also focuses on the impact of management programs and new therapeutic agents and protocols on patient perspectives such as quality of life, adherence and satisfaction. The manuscript management system is completely online and includes a very quick and fair peer-review system, which is all easy to use. Visit http://www.dovepress.com/ testimonials.php to read real quotes from published authors. 